\title{
Structural basis of membrane disruption and cellular toxicity by $\alpha$-synuclein oligomers
}

\author{
Giuliana Fusco, ${ }^{1,2}$ Serene W. Chen, ${ }^{1,2}$ Philip T. F. Williamson, ${ }^{3}$ Roberta Cascella, ${ }^{4}$ \\ Michele Perni, ${ }^{1}$ James A. Jarvis, ${ }^{2}$ Cristina Cecchi, ${ }^{4}$ Michele Vendruscolo, ${ }^{1}$ \\ Fabrizio Chiti, ${ }^{4}$ Nunilo Cremades, ${ }^{5}$ Liming Ying, ${ }^{6}$ \\ Christopher M. Dobson, ${ }^{1 *}$ Alfonso De Simone ${ }^{2 *}$
}

\begin{abstract}
Oligomeric species populated during the aggregation process of $\boldsymbol{\alpha}$-synuclein have been linked to neuronal impairment in Parkinson's disease and related neurodegenerative disorders. By using solution and solid-state nuclear magnetic resonance techniques in conjunction with other structural methods, we identified the fundamental characteristics that enable toxic $\alpha$-synuclein oligomers to perturb biological membranes and disrupt cellular function; these include a highly lipophilic element that promotes strong membrane interactions and a structured region that inserts into lipid bilayers and disrupts their integrity. In support of these conclusions, mutations that target the region that promotes strong membrane interactions by $\alpha$-synuclein oligomers suppressed their toxicity in neuroblastoma cells and primary cortical neurons.
\end{abstract}

\footnotetext{
T
} he aggregation of $\alpha$-synuclein $(\alpha S)$ into amyloid fibrils within Lewy bodies is associated with Parkinson's disease and a range of other debilitating neurodegenerative disorders (1-9). The primary pathogenic agents in these conditions are thought to be the oligomeric species populated during the self-assembly of $\alpha \mathrm{S}$, particularly through their aberrant interactions with biological membranes (10-16). Here we investigated two types of stabilized $\alpha \mathrm{S}$ oligomers with considerably different toxicities $(17,18)$, which we designated as type $A^{*}$ and type $B^{*}$. Their fluorescence resonance energy transfer (FRET) signatures (Fig. 1A), as well as other structural those of the previously identified transient forms of nontoxic (type A) and toxic (type B) $\alpha \mathrm{S}$ oligomers (19).

The two oligomeric forms of $\alpha \mathrm{S}$ had similar sizes and morphologies (Fig. 1, B and C), yet exhibited very different abilities to disrupt lipid bilayers. When incubated in vitro with small unilamellar vesicles (SUVs) composed of 1-palmitoyl2-oleoyl-sn-glycero-3-phospho---serine(POPS) lipids, type- $\mathrm{A}^{*}$ oligomers induced only a marginal release of encapsulated calcein molecules, comparable to that induced by $\alpha \mathrm{S}$ monomers and mature fibrils (Fig. 1D). By contrast, type-B* oligomers and biological properties (Fig. 1), closely match induced greater than 10 times more calcein release, indicating that these oligomers generate considerable disruption of acidic lipid bilayers (Fig. 1D). We observed similar loss of membrane integrity in vivo upon incubation of type- $\mathrm{B}^{*} \alpha \mathrm{S}$ oligomers with human neuroblastoma SH-SY5Y cells and rat primary cortical neurons and only marginal effects upon incubation with type- $A^{*}$ oligomers, monomers, and mature fibrils of $\alpha \mathrm{S}$ (Fig. 1, E and G).

The remarkable disruption of synthetic and cellular membranes by type- $\mathrm{B}^{*} \alpha \mathrm{S}$ oligomers, but not type- $A^{*}$ species, was strongly correlated with their ability to generate cellular toxicity. Thus, type- $\mathrm{B}^{\star} \alpha \mathrm{S}$ oligomers-but not monomers, type- $\mathrm{A}^{*}$ oligomers, and $\alpha \mathrm{S}$ fibrils-induced substantial increases of intracellular reactive oxygen species (ROS, fig. S1A) and reduced the mitochondrial activity in neuronal cells [3-(4,5-dimethylthiazol2-yl)-2,5-diphenyltetrazolium bromide (MTT) assay, Fig. $1 \mathrm{~F}$ ]. The cellular damage that type- $\mathrm{B}^{*} \alpha \mathrm{S}$ oligomers induced in this manner reproduces a variety of pathophysiological effects observed in neuronal models of PD that were obtained by inducing pluripotent stem cell-derived neurons from a patient with triplication of the $\alpha \mathrm{S}$ gene (20-22).

To probe the structural properties of the two types of $\alpha$ S oligomers, we used solid-state nuclear magnetic resonance (ssNMR) spectroscopy.

${ }^{1}$ Department of Chemistry University of Cambridge, Cambridge CB2 1EW, UK. 'Department of Life Sciences, Imperial College London, London SW7 2AZ, UK. ${ }^{3}$ Centre for Biological Sciences and Institute for Life Sciences, University of Southampton, Southampton S017 1BJ, UK. ${ }^{4}$ Department of Experimental and Clinical Biomedical Sciences, Section of Biochemistry, University of Florence, 50134 Florence, Italy. ${ }^{5}$ Biocomputation and Complex Systems Physics Institute (BIFI), Joint Unit BIFIInstituto de Química Física "Rocasolano" (Consejo Superior de Investigaciones Científicas), University of Zaragoza, 50018 Zaragoza, Spain. ${ }^{6}$ Molecular Medicine, National Heart and Lung Institute, Imperial College London, London SW7 2AZ, UK. *Corresponding author. Email: cmd44@cam.ac.uk (C.M.D.); adesimon@imperial.ac.uk (A.D.)
Fig. 1. Properties of the two types of $\boldsymbol{\alpha}$ S oligomers. (A) Intermolecular FRET efficiencies from measurements of type- $A^{*}$ (orange) and type- $\mathrm{B}^{*}$ (green) $\alpha \mathrm{S}$ oligomers. (B) Images of the type- $A^{*}$ and type- $B^{*}$ samples of $\alpha$ S oligomers probed by atomic force microscopy (scale bar, $200 \mathrm{~nm}$ ). (C) Sedimentation coefficients (S) of the type- $A^{*}$ (orange) and the type- $B^{*}$ (green) oligomers measured in arbitrary units. (D) In vitro calcein release (\%) from POPS SUVs (see supplementary methods). (E and F) Intracellular calcein-induced fluorescence (E) and mitochondrial activity monitored by the reduction of MTT (F) measured on human neuroblastoma SH-SY5Y cells (filled bars) and rat primary cortical neurons (striped bars) after incubation with the various $\alpha \mathrm{S}$ species. The data in panels (D) to (F) report the mean values \pm standard deviations (see table $\mathrm{S} 2$ for $P$ values). (G) Representative confocal scanning microscopy images of $\mathrm{SH}-\mathrm{SY} 5 \mathrm{Y}$ cells (scale bar, $30 \mu \mathrm{m}$ ), showing intracellular calcein-induced fluorescence after incubation with the various $\alpha$ s species.
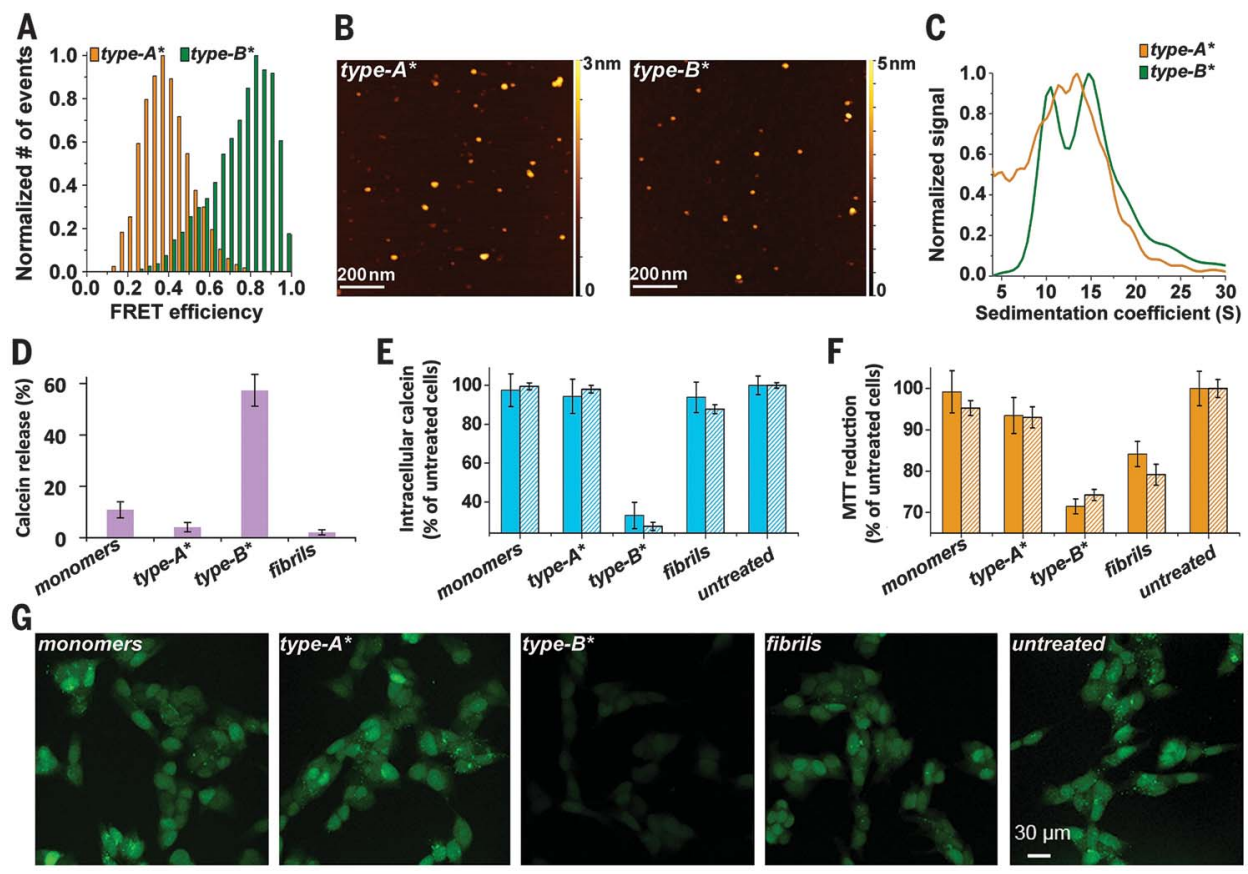
Correlations between carbon atoms of residues located in rigid regions of the oligomers were detected using ${ }^{13} \mathrm{C}-{ }^{13} \mathrm{C}$ dipolar-assisted rotational resonance (DARR) correlation spectra, measured using magic angle spinning (MAS). ${ }^{13} \mathrm{C}-{ }^{3} \mathrm{C}$ DARR spectra of type- $\mathrm{A}^{*}$ and type- $\mathrm{B}^{\star}$ oligomers revealed fundamental differences in the structural nature of the two species (Fig. 2A), with considerable $\beta$-sheet content identified in the rigid regions of the type- $\mathrm{B}^{*}$ oligomers and negligible secondarystructure content associated with the rigid regions of type-A* oligomers (Fig. 2A and fig. S2). The assignment of the ${ }^{13} \mathrm{C}-{ }^{13} \mathrm{C}$ DARR peaks was performed using an approach (23-25) that combines information from solution-state chemical exchange saturation transfer (CEST) $(23,26,27)$ (see supplementary methods and fig. S3) with known assignments of fibrillar (5) and monomeric (25) states of $\alpha \mathrm{S}$. This approach revealed that the resonances in the ${ }^{13} \mathrm{C}-{ }^{13} \mathrm{C}$ DARR spectra of the two types of oligomers belong to specific regions spanning residues 3 to 36 and 70 to 88 in type- $A^{*}$ and type- $\mathrm{B}^{*}$ species, respectively (table S1).

Highly mobile regions of the two forms of $\alpha \mathrm{S}$ oligomers were detected by using insensitive nuclei enhanced by polarization transfer (INEPT) measurements in MAS ssNMR (Fig. 2B). Both types of oligomers contained a large number of highly mobile residues (45 in type $\mathrm{A}^{*}$ and 67 in type $\mathrm{B}^{*}$, table $\mathrm{S} 1$ ), whose resonances overlapped with those in the ${ }^{1} \mathrm{H}_{-}{ }^{13} \mathrm{C}$ heteronuclear singlequantum coherence spectra of disordered monomeric $\alpha \mathrm{S}$ in solution (fig. S4). Although both oligomeric species possessed a flexible $\mathrm{C}$-terminal region (43 and 40 highly mobile C-terminal residues for type $\mathrm{A}^{*}$ and type $\mathrm{B}^{*}$, respectively), the $\mathrm{N}$-terminal region of $\alpha \mathrm{S}$ was highly dynamic only in the type- $\mathrm{B}^{*}$ oligomers ( 0 and 26 highly mobile $\mathrm{N}$-terminal residues for type $\mathrm{A}^{*}$ and type $\mathrm{B}^{*}$, respectively). Furthermore, the mobile regions of the type- $\mathrm{A}^{*} \alpha \mathrm{S}$ oligomers also included residues from the nonamyloid- $\beta$ component (NAC) region (Fig. 2B), a finding consistent with the low FRET efficiency observed for the type-A* oligomers relative to that for the type- $\mathrm{B}^{*}$ oligomers when the fluorophores were attached to residue 90 , itself located in the NAC region (Fig. 1A).

The ssNMR characterization of the two types of $\alpha \mathrm{S}$ oligomers was supported by Fourier transform infrared spectroscopy measurements (fig. S5), which showed signals characteristic of both random-coil and $\beta$-sheet structure in the case of the type- $\mathrm{B}^{*} \alpha \mathrm{S}$ oligomers but indicated a predominantly unstructured conformation for the type- $A^{*}$ species. Further indication of the association with the core of the oligomers in type- $\mathrm{A}^{*}$ and type- $\mathrm{B}^{*}$ species was provided by CEST experiments (fig. S3). Type- $A^{*}$ oligomers revealed extensive saturation in the $40 \mathrm{~N}$-terminal residues of the protein, indicating strong association of this region with the core of the oligomers. For type- $\mathrm{B}^{*}$ oligomers, CEST experiments showed no saturation associated with either the N- or C- terminal regions of the protein or with residues of three of the four major hydrophobic segments of the $\alpha \mathrm{S}$ sequence (residues 36 to 41,47 to 56 , and 88 to $95)$, in contrast to the strong saturation observed
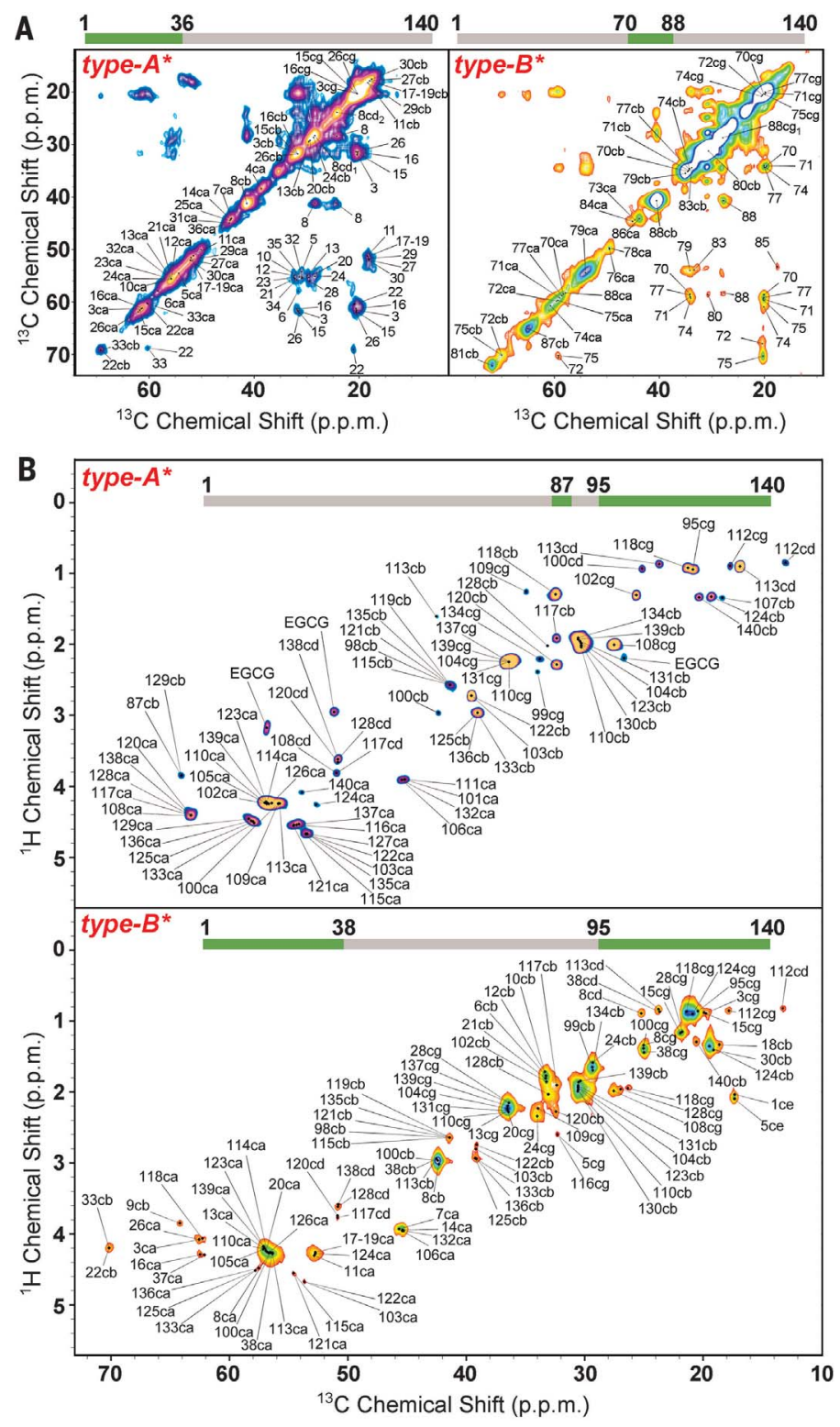

Fig. 2. MAS ssNMR spectra of $\alpha S$ oligomers. (A) ${ }^{13} \mathrm{C}-{ }^{13} \mathrm{C}$ DARR correlation spectra (aliphatic regions) of type- $A^{*}$ and type- $B^{*} \alpha S$ oligomers (left and right panels, respectively). (B) ${ }^{1} \mathrm{H}^{13} \mathrm{C}$ correlations by means of INEPT transfer. The labels $\mathrm{ca}, \mathrm{cb}, \mathrm{cg}, \mathrm{cd}$, and ce indicate $C^{\alpha}, C^{\beta}, C^{\gamma}$, $\mathrm{C}^{\delta}$, and $\mathrm{C}^{\varepsilon}$ atoms, respectively. The regions of the protein sequence detected in the spectra are noted by residue number and highlighted in green in the bars above the spectra. p.p.m., parts per million.

for the hydrophobic segment of residues 70 to 79. The identification of highly dynamic regions in the two types of $\alpha \mathrm{S}$ oligomers was consistent with a dot blot analysis carried out using a primary antibody that binds the $\mathrm{N}$-terminal region of $\alpha \mathrm{S}$. This indicated that the $\mathrm{N}$-terminal region is more accessible in type- $\mathrm{B}^{*}$ than in type- $\mathrm{A}^{*}$ species (fig. S6). By contrast, dot blot analysis carried out using a primary antibody targeting the C-terminal region of $\alpha \mathrm{S}$ indicated that the $\mathrm{C}$ terminus is accessible to a similar extent in both types of oligomers (fig. S6).

We next probed the interaction of the two types of $\alpha$ S oligomers with SUVs composed of DOPE:DOPS:DOPC lipids in a ratio of 5:3:2 (see supplementary methods), which are good mimics of synaptic vesicles for composition and physical properties $(24,25)$ (DOPE, 1,2-dioleoyl-sn-glycero3-phosphoethanolamine; DOPS, 1,2-dioleoyl-snglycero-3-phospho-L-serine; DOPC, 1,2-dioleoyl-snglycero-3-phosphocholine). Fluorescence correlation spectroscopy (FCS) in combination with confocal microscopy showed that both types of oligomers bind the SUVs with high affinity, with the type- $B^{*}$ oligomers binding more strongly relative to the type- $A^{*}$ species (Fig. 3A and fig. S7). Type- $B^{*} \alpha S$ oligomers also colocalized with the plasma membrane of primary cortical neurons (Fig. 3B).

To characterize the levels of membrane insertion of the two types of $\alpha \mathrm{S}$ oligomers, we carried out paramagnetic relaxation enhancement (PRE) experiments using MAS ssNMR, in which small quantities of lipid molecules labeled with a paramagnetic center $(\mathrm{PC})$ were incorporated into the bilayers $(24,25)$. When the PC was located in the hydrophilic head groups of the lipid molecules (see supplementary methods), selective quenching of a number of ${ }^{13} \mathrm{C}-{ }^{13} \mathrm{C}$ DARR resonances was observed in the spectra of both types of $\alpha$ S oligomers (fig. S8). This indicates, in both cases, strong interactions with the membrane surface. By contrast, when the PC was positioned 
Fig. 3. Interactions of $\alpha S$ oligomers with lipid bilayers. (A) Diffusion times, $\tau_{D}$, from FCS experiments of type- $A^{*}$ and type- $B^{*} \alpha$ S oligomers in the presence of variable quantities of SUVS composed of DOPE:DOPS:DOPC. The $\tau_{D}$ values and errors are derived from the fitting of the autocorrelation function (see supplementary methods and fig. S7). (B) Representative confocal scanning microscopy images of basal, median, and apical planes and their combination (fourth column) of primary cortical neurons after 15 min incubation with type- $B^{*} \alpha S$ oligomers. Red and green fluorescence indicates the cell membrane and the $\alpha \mathrm{S}$ oligomers, respectively. Lower panels correspond to magnifications of the sections indicated with a white square in the upper panels. (C) PRE effects measured using MAS ssNMR for type- $A^{*}$ (left) and type- $B^{*}$ (right) $\alpha$ S oligomers using SUVs with a PC at the position of carbon 16 of the lipid chain. ${ }^{13} \mathrm{C}-{ }^{13} \mathrm{C}$ DARR spectra measured in the presence and absence of the paramagnetic-labeled lipids are shown in blue and red, respectively. (D) ${ }^{13} \mathrm{C}^{13} \mathrm{C}$ DARR spectra of isolated type- $A^{*}$ (left) and type- $B^{*}$ (right) $\alpha$ S oligomers (red) compared with spectra measured at $-19^{\circ} \mathrm{C}$ in the presence of DOPE:DOPS:DOPC SUVs (green) and of monomeric $\alpha S$ bound to the same type of vesicles (24) (black contour lines). The arrows identify the signals of residues in the $\alpha$ S oligomers arising from the membrane-bound protein regions. In the case of type- $B^{*} \alpha S$ oligomers, these regions were assigned based on a previous study of the monomeric state of $\alpha \mathrm{S}$ bound to SUVS (24). E, glutamic acid; Q, glutamine; K, lysine; V, valine; A, alanine; $T$, threonine. (E and $\mathbf{F}$ ) Schematic representation of the binding of type- $A^{*}$ and type- $B^{*}$ with biological membranes. (E) Type- $A^{*} \alpha S$ oligomers (left) are mainly disordered and bind exclusively to the membrane surface.

C

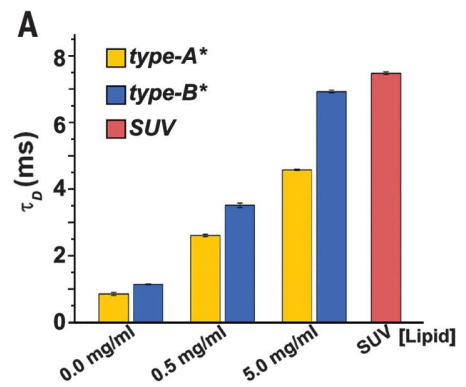

B
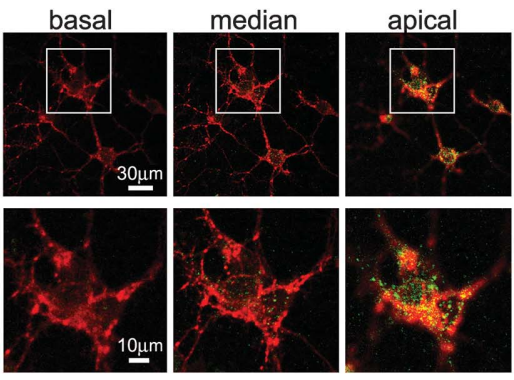

all planes

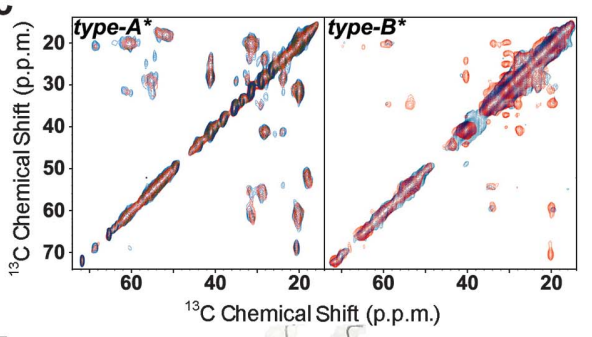

D
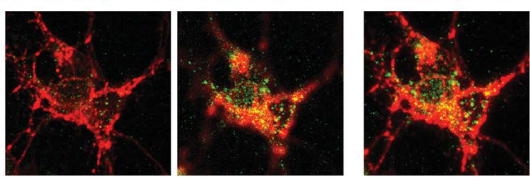

E

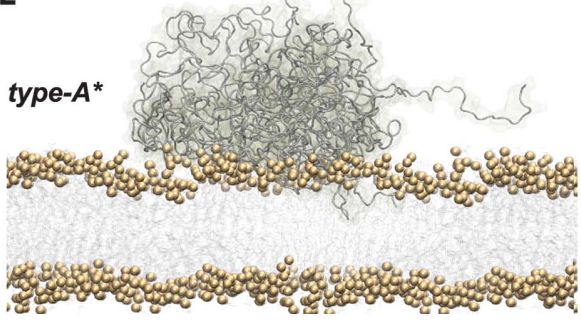

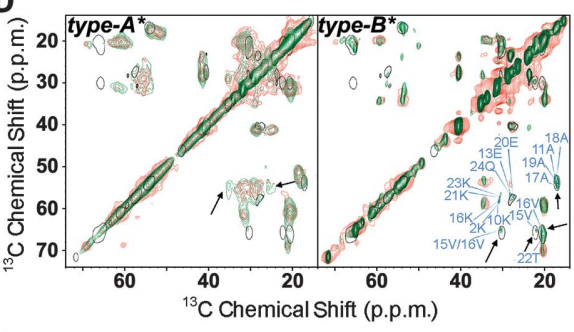

$\mathbf{F}$

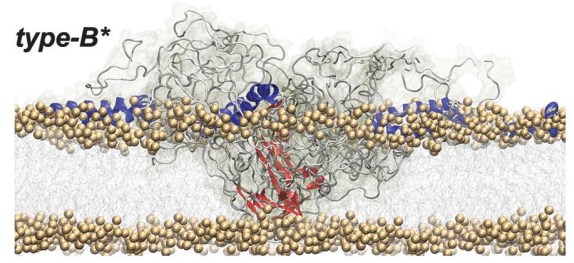

(F) Type- $B^{*} \alpha S$ oligomers feature both structured (red) and disordered (gray) regions and bind the surfaces of the lipid bilayers via the folding of $N$-terminal regions into amphipathic $\alpha$-helices (blue) upon membrane binding. The rigid regions of type- $B^{*} \alpha S$ oligomers, which are rich in $\beta$-sheet structure, insert into the lipid bilayers, thereby disrupting their integrity. within the interior of the lipid bilayer, enhanced relaxation of ${ }^{13} \mathrm{C}-{ }^{13} \mathrm{C}$ DARR resonances was observed only for the type- $\mathrm{B}^{*}$ oligomers (Fig. 3C); this suggests that type- $\mathrm{B}^{*}$ oligomers can insert into the hydrophobic interior of the lipid bilayer, whereas the type-A* oligomers remain bound exclusively to the membrane surface (Fig. 3, E and $\mathrm{F}$ ). In addition, the ${ }^{1} \mathrm{H}_{-}{ }^{13} \mathrm{C}$ INEPT spectra of the type- $\mathrm{B}^{*}$ oligomers showed peak broadening only in PRE experiments performed when the PC was located in the lipid head groups (fig. S9); this suggests that dynamic regions of type- $\mathrm{B}^{*}$ oligomers do not insert in the hydrophobic interior of the lipid bilayer.

To characterize the structural nature of the regions of $\alpha \mathrm{S}$ that are tightly bound to the lipid bilayers in the two types of oligomers, we measured ${ }^{13} \mathrm{C}-{ }^{13} \mathrm{C}$ DARR spectra at $-19^{\circ} \mathrm{C}$ (Fig. 3D). This condition enhances the ${ }^{13} \mathrm{C}_{-13}{ }^{13} \mathrm{C}$ DARR signals of the protein regions that are tightly bound with the surface of this type of acidic lipid membrane $(24,25)$. In the case of the type-A* oligomers, we observed an additional set of peaks whose resonances are characteristic of lysine residues, which are abundant in the region of residues 1 to 97 of the $\alpha$ S sequence. The broad linewidths of these resonances, however, indicated the absence of a defined structural motif of type-A* oligomers that is tightly bound to the membrane. By contrast, for the type- $\mathrm{B}^{*}$ species, we observed a set of additional resonances that closely matched those of the N-terminal 25 residues of monomeric $\alpha \mathrm{S}$ bound to SUVs in an amphipathic $\alpha$-helical conformation $(12,25,28)$. Thus, the N-terminal region in the toxic oligomers is involved in promoting strong membrane interactions.

We next examined the effects that mutations in the $\mathrm{N}$-terminal region of $\alpha \mathrm{S}$ exert on the ability of type- $\mathrm{B}^{*}$ oligomers to disrupt biological membranes and induce cellular toxicity. The $\alpha \mathrm{S}$ variant $\alpha \mathrm{S}_{\mathrm{A} 30 \mathrm{P}}$ (A30P, alanine-to-proline substitution at residue 30 ) is associated with an early onset form of Parkinson's disease and reduces the membrane affinity of the N-terminal region of $\alpha \mathrm{S}(24)$. We also examined a truncated form of the protein lacking residues 2 to $9\left(\alpha \mathrm{S}_{\Delta 2-9}\right)$, a key region involved in anchoring the $\mathrm{N}$-terminal region of $\alpha \mathrm{S}$ to lipid membranes $(25,29)$. In vitro measurements of calcein release from POPS SUVs showed intermediate and negligible levels of membrane disruption by type-B* oligomers formed from $\alpha S_{A 30 P}$ and $\alpha S_{\Delta 2-9}$, respectively, compared to the effects of wild-type $\alpha \mathrm{S}\left(\alpha \mathrm{S}_{\mathrm{WT}}\right)$ type- $\mathrm{B}^{*}$ oligomers (fig. S10A). Similar trends were observed in the disruption of cellular membranes by these species (fig. S10B) and in their colocalization with the plasma membrane (fig. S10, D and E). The partial and total impairment of the binding and disruption of cellular membranes by type- $\mathrm{B}^{*}$ oligomers of $\alpha \mathrm{S}_{\mathrm{A} 30 \mathrm{P}}$ and $\alpha \mathrm{S}_{\Delta 2-9}$, respectively, correlated highly with the levels of cellular toxicity generated by these species (MTT in fig. S1OC and ROS in fig. S1B).

It is well established that the toxic forms of amyloid-related aggregates of many proteins bind to membranes and disrupt cellular function $(1,14)$. Here we have identified specific structural elements of $\alpha \mathrm{S}$ oligomers that give rise to cellular toxicity by disrupting the integrity of biological membranes (Fig. 3F and fig. S11). In primary neurons and astrocytes, this leads to an increase in the amounts of basal intracellular $\mathrm{Ca}^{2+}$ and of ROS and, consequently, a loss of cellular viability $(20,21)$. The first structural element is an exposed highly lipophilic region of the protein in the oligomers that promotes strong interactions with the membrane surface. The second is a rigid oligomeric core that is rich in $\beta$-sheet structure and is able to insert into the lipid bilayer and disrupt the membrane integrity. These conclusions are strongly supported by the introduction of specific mutations that partially (A30P) or completely ( $\Delta 2-9)$ suppress the membrane affinity of the $\mathrm{N}$-terminal sequence of the protein. 
This suggests that the ability of the accessible $\mathrm{N}$-terminal region of $\alpha \mathrm{S}$ to bind strongly to lipid bilayers is a vital step in enabling oligomers of this protein to disrupt cellular membranes and, consequently, to induce neuronal toxicity.

\section{REFERENCES AND NOTES}

1. F. Chiti, C. M. Dobson, Annu. Rev. Biochem. 86, 27-68 (2017).

2. V. N. Uversky, D. Eliezer, Curr. Protein Pept. Sci. 10, 483-499 (2009).

3. E. S. Luth, I. G. Stavrovskaya, T. Bartels, B. S. Kristal, D. J. Selkoe, J. Biol. Chem. 289, 21490-21507 (2014)

4. H. A. Lashuel, C. R. Overk, A. Oueslati, E. Masliah, Nat. Rev. Neurosci. 14, 38-48 (2013)

5. M. D. Tuttle et al., Nat. Struct. Mol. Biol. 23, 409-415 (2016).

6. M. Vilar et al., Proc. Natl. Acad. Sci. U.S.A. 105, 8637-8642 (2008).

7. J. A. Rodriguez et al., Nature 525, 486-490 (2015).

8. L. Bousset et al., Nat. Commun. 4, 2575 (2013).

9. G. Comellas, L. R. Lemkau, D. H. Zhou, J. M. George, C. M. Rienstra, J. Am. Chem. Soc. 134, 5090-5099 (2012).

10. B. Winner et al., Proc. Natl. Acad. Sci. U.S.A. 108, 4194-4199 (2011).

11. M. Grey, S. Linse, H. Nilsson, P. Brundin, E. Sparr, J. Parkinsons Dis. 1. 359-371 (2011)
12. N. Lorenzen, L. Lemminger, J. N. Pedersen, S. B. Nielsen, D. E. Otzen, FEBS Lett. 588, 497-502 (2014)

13. K. M. Danzer et al., J. Neurosci. 27, 9220-9232 (2007).

14. S. Campioni et al., Nat. Chem. Biol. 6, 140-147 (2010).

15. P. K. Auluck, G. Caraveo, S. Lindquist, Annu. Rev. Cell Dev. Biol. 26, 211-233 (2010)

16. J. H. Soper, V. Kehm, C. G. Burd, V. A. Bankaitis, V. M. Lee, J. Mol. Neurosci. 43, 391-405 (2011).

17. S. W. Chen et al., Proc. Natl. Acad. Sci. U.S.A. 112, E1994-E2003 (2015).

18. D. E. Ehrnhoefer et al., Nat. Struct. Mol. Biol. 15, 558-566 (2008).

19. N. Cremades et al., Cell 149, 1048-1059 (2012)

20. P. R. Angelova et al., J. Cell Sci. 129, 1792-1801 (2016).

21. E. Deas et al., Antioxid. Redox Signal. 24, 376-391 (2016).

22. M. J. Devine et al., Nat. Commun. 2, 440 (2011).

23. G. Fusco et al., Nat. Commun. 5, 3827 (2014).

24. G. Fusco et al., Nat. Commun. 7, 12563 (2016).

25. G. Fusco et al., Sci. Rep. 6, 27125 (2016).

26. P. Vallurupalli, G. Bouvignies, L. E. Kay, J. Am. Chem. Soc. 134, 8148-8161 (2012).

27. J. Milojevic, V. Esposito, R. Das, G. Melacini, J. Am. Chem. Soc. 129, 4282-4290 (2007)

28. C. R. Bodner, C. M. Dobson, A. Bax, J. Mol. Biol. 390, 775-790 (2009).

29. K. Vamvaca, M. J. Volles, P. T. Lansbury Jr., J. Mol. Biol. 389 413-424 (2009)

\section{ACKNOWLEDGMENTS}

This research was supported by Parkinson's UK (G-1508 to G.F. M.V., C.M.D., and A.D.); the UK Medical Research Council (MR/N000676/1 to G.F., M.V., C.M.D., and A.D.); the Wellcome Trust (104933/Z/14/Z to A.D.); the Leverhulme Trust (RPG-2015-350 to A.D. and RPG-2015-345 to L.Y.); the British Heart Foundation (PG/14/93/31237 to A.D. and PG/11/81/29130 to L.Y.); the UK Biotechnology and Biological Sciences Research Council (BB/M023923/1 to A.D. and BB/G00594X/1 to L.Y.); the Agency of Science, Technology and Research of Singapore (to S.W.C.); the Ministry of Economy, Industry, and Competitiveness of Spain (MINECO RYC-2012-12068 and MINECO/FEDER EU BFU2015-64119-P to N.C.); the Regione Toscana (SUPREMAL to F.C., C.C., and R.C.); the University of Florence (Fondi di Ateneo to F.C. and C.C.); and the Centre for Misfolding Diseases of the University of Cambridge. The data supporting the findings of this study are available within the article and supplementary materials.

\section{SUPPLEMENTARY MATERIALS}

www.sciencemag.org/content/358/6369/1440/suppl/DC1 Materials and Methods

Figs. S1 to S12

Tables S1 to S2

References (30-44)

9 May 2017; resubmitted 17 September 2017

Accepted 14 November 2017

$10.1126 /$ science aan6160 


\section{Science}

\section{Structural basis of membrane disruption and cellular toxicity by $\alpha$-synuclein oligomers}

Giuliana Fusco, Serene W. Chen, Philip T. F. Williamson, Roberta Cascella, Michele Perni, James A. Jarvis, Cristina Cecchi, Michele Vendruscolo, Fabrizio Chiti, Nunilo Cremades, Liming Ying, Christopher M. Dobson and Alfonso De Simone

Science 358 (6369), 1440-1443.

DOI: $10.1126 /$ science.aan6160

\section{A structural look at $\alpha$-synuclein oligomers}

Fibrillar aggregates of the protein $\alpha$-synuclein $(\alpha S)$ are the major constituents of Lewy bodies in Parkinson's disease. However, small oligomers that accumulate during the process of fibril formation are thought to cause the neuronal toxicity associated with the onset and progression of Parkinson's disease. Little is known about the detailed structural properties of $\alpha$ S oligomers and the molecular mechanisms that lead to their toxicity. Fusco et al. report the structural characterization of two forms of $\alpha$ S oligomers, which elucidates the fundamental structural elements giving rise to neuronal toxicity.

Science, this issue p. 1440

ARTICLE TOOLS

SUPPLEMENTARY MATERIALS

RELATED
CONTENT

REFERENCES

PERMISSIONS http://science.sciencemag.org/content/358/6369/1440

http://science.sciencemag.org/content/suppl/2017/12/13/358.6369.1440.DC1

http://stm.sciencemag.org/content/scitransmed/8/368/368ra174.full

http://stm.sciencemag.org/content/scitransmed/8/367/367ra170.full http://stm.sciencemag.org/content/scitransmed/8/342/342ra78.full http://stm.sciencemag.org/content/scitransmed/4/163/163ra156.full

This article cites 43 articles, 6 of which you can access for free http://science.sciencemag.org/content/358/6369/1440\#BIBL

http://www.sciencemag.org/help/reprints-and-permissions

Use of this article is subject to the Terms of Service

Science (print ISSN 0036-8075; online ISSN 1095-9203) is published by the American Association for the Advancement of Science, 1200 New York Avenue NW, Washington, DC 20005. The title Science is a registered trademark of AAAS.

Copyright @ 2017 The Authors, some rights reserved; exclusive licensee American Association for the Advancement of Science. No claim to original U.S. Government Works 